\title{
A Theory of Summability on a Space of Generalized Functions
}

\author{
Khaula Naeem Khan ${ }^{1}$ and Wilson Lamb ${ }^{2}$ \\ ${ }^{1}$ Centre of Advanced Mathematics and Physics, National University of Sciences and Technology, Islamabad 44000, Pakistan \\ ${ }^{2}$ Department of Mathematics and Statistics, University of Strathclyde, 26 Richmond Street, Livingston Tower, Glasgow G1 1XH, UK
}

Correspondence should be addressed to Khaula Naeem Khan; khaulanaeem@gmail.com

Received 30 January 2013; Revised 1 July 2013; Accepted 2 July 2013

Academic Editor: Alberto Fiorenza

Copyright (c) 2013 K. N. Khan and W. Lamb. This is an open access article distributed under the Creative Commons Attribution License, which permits unrestricted use, distribution, and reproduction in any medium, provided the original work is properly cited.

\begin{abstract}
A theory of summability of orthonormal sets is introduced in multinormed spaces. The approach which is presented caters for infinite sets $\left\{\phi_{j}\right\}_{j \in J}$, where the index set $J$ may be uncountable, and is applied to obtain convergence results in appropriate spaces of test functions and corresponding spaces of generalized functions. These spaces are constructed in a systematic manner that relies heavily on properties of orthonormal bases in Hilbert spaces. A space of almost-periodic generalized functions, in which each generalized function can be expanded in terms of an uncountable basis of exponential functions, is obtained as a special case of our theory.
\end{abstract}

\section{Introduction}

In recent papers $[1,2]$, we investigated the behaviour of certain fractional differential and integral operators on appropriately defined spaces of generalized functions. In each paper, a procedure for producing spaces of test functions and generalized functions, pioneered by Zemanian [3, Chapter 9], played a crucial role. Central to this procedure is the theory of complete orthonormal sequences in $L^{2}$-type spaces. Given a complete orthonormal sequence $\left\{\psi_{n}\right\}_{n=0}^{\infty}$ of eigenfunctions of a suitably restricted symmetric differential operator $T$ in $L^{2}(I)$, where $I$ is an interval in $\mathbb{R}$, corresponding spaces of test functions $\mathscr{A}(=\mathscr{A}(I))$ and generalized functions $\mathscr{A}^{\prime}$ (= $\left.\mathscr{A}^{\prime}(I)\right)$ are obtained in a systematic and constructive manner.

In the theory developed by Zemanian, the operator $T$ : $L^{2}(I) \supset D(T) \rightarrow L^{2}(I)$ is a differential expression of the form

$$
T=\theta_{0} D^{n_{1}} \theta_{1} D^{n_{2}} \cdots D^{n_{v}} \theta_{v},
$$

where $D=d / d x$, the $n_{k}$ are positive integers, and the $\theta_{k}$ are smooth functions on $I$. Moreover, it is assumed that $T$ satisfies the (symmetry) condition

$$
T=\overline{\theta_{\nu}}(-D)^{n_{\nu}} \cdots(-D)^{n_{2}} \overline{\theta_{1}}(-D)^{n_{1}} \overline{\theta_{0}}
$$

and has smooth eigenfunctions $\left\{\psi_{n}\right\}_{n=0}^{\infty}$ that form a complete orthonormal basis for $L^{2}(I)$ and for which the corresponding real eigenvalues $\left\{\lambda_{n}\right\}_{n=0}^{\infty}$ are such that $\left|\lambda_{n}\right| \rightarrow \infty$ as $n \rightarrow \infty$. The domain $D(T)$ of the operator $T$ is

$$
\begin{aligned}
D(T):=\{\phi & \in C^{\infty}(I): T^{k} \phi \in L^{2}(I),\left(T^{k} \phi, \psi_{n}\right)_{2} \\
& \left.=\left(\phi, T^{k} \psi_{n}\right)_{2}, \forall k, n=0,1, \ldots\right\},
\end{aligned}
$$

where $T^{0}$ is the identity operator on $L^{2}(I)$; see [3, Chapter 9]. The space of test functions is then taken to be the vector space $D(T)$ equipped with the topology generated by the countable multinorm $\left\{\beta_{k}\right\}_{k=0}^{\infty}$, where

$$
\beta_{k}(\phi):=\left\|T^{k} \phi\right\|_{2}<\infty, \quad \forall k=0,1,2, \ldots
$$

The resulting topological vector space can be shown to be a Fréchet space and is denoted by $\mathscr{A}$. Note that $D(T)$ is a dense subspace of $L^{2}(I)$, since $C_{0}^{\infty}(I) \subset D(T)$, and that each eigenfunction $\psi_{n} \in D(T)$. The space of generalized functions $\mathscr{A}^{\prime}$ is the dual of $\mathscr{A}$ equipped with the weak* topology generated by the multinorm $\left\{\alpha_{\phi}\right\}$, where

$$
\alpha_{\phi}(f)=|(f, \phi)|, \quad f \in \mathscr{A}^{\prime}, \phi \in \mathscr{A},
$$

where $(f, \phi)$ is the number assigned to each $\phi \in \mathscr{A}$ by $f \in \mathscr{A}^{\prime}$. Since $\mathscr{A}^{\prime}$ is the dual of a Fréchet space, it is sequentially complete with respect to the weak ${ }^{*}$ topology. 
Also, by identifying functions $\varphi \in L^{2}(I)$ with regular generalized functions $\widetilde{\varphi} \in \mathscr{A}^{\prime}$ via

$$
(\widetilde{\varphi}, \phi):=(\phi, \varphi)_{2}=\int_{I} \phi(x) \overline{\varphi(x)} d x, \quad \phi \in \mathscr{A},
$$

it can be shown that $L^{2}(I)$ is continuously imbedded in $\mathscr{A}^{\prime}$. Consequently, we can write

$$
\mathscr{A} \hookrightarrow L^{2}(I) \hookrightarrow \mathscr{A}^{\prime},
$$

where $\hookrightarrow$ represents a continuous embedding. Results on the weak $^{*}$ convergence of eigenfunction expansions of generalized functions in the space $\mathscr{A}^{\prime}$ are given in [3, Chapter 9], where it is shown that

$$
f=\sum_{n=0}^{\infty}\left(f, \psi_{n}\right) \widetilde{\psi}_{n}, \quad \forall f \in \mathscr{A}^{\prime} .
$$

It should be noted that the Zemanian theory of eigenfunction expansions has been extended in a number of ways since its introduction; see, for example, [4-7]. In particular, in [5] the differential operator that is used to construct the Fréchet space $\mathscr{A}$ is replaced by any self-adjoint operator $T$ defined on an arbitrary separable Hilbert space $H$. The space of "testfunctions" is then taken to be the Fréchet space

$$
D_{\infty}:=\bigcap_{k=1}^{\infty} D\left(T^{k}\right)
$$

in which convergence is defined via the countable multinorm $\left\{\beta_{k}\right\}_{k=0}^{\infty}, \beta_{k}(\phi):=\left\|T^{k} \phi\right\|_{H}, \phi \in D_{\infty}$. The corresponding space of "generalized functions" is given by $D_{\infty}^{\prime}$ equipped with the weak ${ }^{*}$ topology. The Zemanian results arise as the special case when $H=L^{2}(I)$ and $T$ is a self-adjoint differential operator whose spectrum contains only eigenvalues; see [5, Remark 3.5]. One of the aims of the present paper is to demonstrate that a Zemanian-type theory of eigenfunction expansions, but possibly involving an uncountable orthonormal basis, can be developed in the more general setting of an arbitrary Hilbert space. This theory will be based on an extension to complete multinormed spaces of the concept of summability in Banach spaces. It is clear that a self-adjoint operator on a separable Hilbert space cannot have an uncountable basis, as all orthonormal bases in a separable Hilbert space are countable; see [8, p.255]. Consequently, in the sequel, we do not assume that that the Hilbert space $H$ is separable.

In elementary analysis, the sum of an infinite sequence of terms $\phi_{1}, \phi_{2}, \ldots$ in some topological vector space exists if the limit of the corresponding sequence of partial sums exists. To deal with the case of summations of a family of elements $\left\{\phi_{j}\right\}_{j \in J}$ over a general index set $J$, which could be uncountable, requires the notion of summability. There is a well-established theory of summability in a Banach space setting; see, for example, [9, Chapter 6] and [10, Chapter 4]. However, as far as we are aware, a similar theory has not been presented within the framework of a complete multinormed space. Consequently, we begin in Section 2 by giving an appropriate definition of summability in this more general setting and verify that the definition satisfies all the important computational rules.

In Section 3, we establish that the definition of summability given in Section 2 can be used to extend the Zemanian theory of eigenfunction expansions of generalized functions to the case where the orthonormal basis may be uncountable. As the spaces $L^{2}(I)$ favoured by Zemanian are separable, we work within the more general framework of a Hilbert space $H$, which need not be separable, and use results from [5]. As a specific example of an uncountable basis, we go on in Section 4 to examine the nonseparable Hilbert space of $L^{2}$ almost-periodic functions and show how the theory developed in the previous sections leads nicely to eigenfunction expansions for a class of almost-periodic generalized functions.

\section{Infinite Sums in Multinormed Spaces}

A multinormed space is a topological vector space in which the topology is defined by a collection of seminorms. Let $X$ be a vector space and let $S=\left\{\gamma_{\lambda}\right\}_{\lambda \in \Lambda}$ be a separating collection of seminorms on $X$ for some index set $\Lambda$. If $S$ is countable, then the resulting topological vector space, which we will continue to denote simply by $X$, is a countably multinormed space, which, if complete, is a Fréchet space; see [11, p.132].

Let $\left\{\phi_{j}\right\}_{j \in J}$ be an indexed family in $X$ and let $\mathscr{F}$ denote the collection of all finite subsets of $J$. If we use the binary operation $\subseteq$ to define a partial order on $\mathscr{F}$, then $\mathscr{F}$ becomes a directed set. Associated with $\left\{\phi_{j}\right\}_{j \in J}$ is the indexed set of finite partial sums, given by

$$
S_{K}:=\sum_{j \in K} \phi_{j}, \quad K \in \mathscr{F} .
$$

The mapping $K \rightarrow S_{K}$ from $\mathscr{F} \rightarrow X$, is then a net in $X$ and so we can use the theory associated with nets to provide a definition of the sum $\sum_{j \in J} \phi_{j}$, in terms of a limit of finite partial sums, which is independent of the way in which the terms $\phi_{j}$ in the sum are ordered.

Definition 1. Let $X$ be a complete multinormed space with multinorm $\left\{\gamma_{\lambda}\right\}_{\lambda \in \Lambda}$. The vectors $\left\{\phi_{j}\right\}_{j \in J} \subset X$ are said to be summable to $\phi \in X$, or, equivalently, the unordered sum of the indexed set $\left\{\phi_{j}\right\}_{j \in J}$ converges to $\phi \in X$, if, for each $\epsilon>0$ and $\lambda \in \Lambda$, there exists $J_{0}\left(=J_{0}(\epsilon, \lambda)\right) \in \mathscr{F}$ such that, for all $K \in \mathscr{F}$ with $J_{0} \subseteq K$,

$$
\gamma_{\lambda}\left(\sum_{j \in K} \phi_{j}-\phi\right)<\epsilon .
$$

In this case, we write $\sum_{j \in J} \phi_{j}=\phi$ and say that the unordered sum converges unconditionally to $\phi$.

In the subsequent work, the phrases " $\sum_{j} \phi_{j}$ converges to $\phi$," "the vectors $\left\{\phi_{j}\right\}_{j \in J}$ are summable to $\phi$," and " $\left\{\phi_{j}\right\}_{j \in J}$ is summable to $\phi$ " will all be used when $\sum_{j \in J} \phi_{j}=\phi$ in $X$.

Lemma 2. If $\sum_{j \in J} \phi_{j}=\phi$, then $\phi$ is unique.

Proof. Let $\sum_{j \in J} \phi_{j}=\phi$ and also let $\sum_{j \in J} \phi_{j}=\psi$ in $X$. Then, from Definition 1, for each $\epsilon>0$ and $\lambda \in \Lambda$, there exists 
$J_{0}\left(=J_{0}(\epsilon, \lambda)\right) \in \mathscr{F}$ such that whenever $K \in \mathscr{F}$ contains $J_{0}$, we have

$$
\gamma_{\lambda}\left(\sum_{j \in K} \phi_{j}-\phi\right)<\frac{\epsilon}{2}
$$

Similarly, for $\epsilon>0$ and $\lambda \epsilon \Lambda$, there exists $K_{0}\left(=K_{0}(\epsilon, \lambda)\right) \epsilon$ $\mathscr{F}$ such that whenever $M \in \mathscr{F}$ contains $K_{0}$,

$$
\gamma_{\lambda}\left(\sum_{j \in M} \psi_{j}-\psi\right)<\frac{\epsilon}{2}
$$

Now, setting $L_{0}=K_{0} \cup J_{0}$, let $L$ be any set from $\mathscr{F}$ containing $L_{0}$. Then $L$ contains both $J_{0}$ and $K_{0}$. Hence, from (12) and (13), we obtain

$$
\begin{aligned}
\gamma_{\lambda}(\phi-\psi) & =\gamma_{\lambda}\left(\sum_{j \in L} \phi_{j}-\psi-\sum_{j \in L} \phi_{j}+\phi\right) \\
& \leq \gamma_{\lambda}\left(\sum_{j \in L} \phi_{j}-\psi\right)+\gamma_{\lambda}\left(\sum_{j \in L} \phi_{j}-\phi\right) \\
& <\frac{\epsilon}{2}+\frac{\epsilon}{2}=\epsilon,
\end{aligned}
$$

and so

$$
\gamma_{\lambda}(\phi-\psi)=0
$$

Since this holds for all $\lambda$ and $\left\{\gamma_{\lambda}\right\}_{\lambda \in \Lambda}$ is a multinorm, it follows that $\phi=\psi$.

Lemma 3. Let $\sum_{j \in J} \phi_{j}=\phi$ and let $\sum_{j \in J} \psi_{j}=\psi$ in $X$. Then

(i) $\sum_{j \in J}\left(\phi_{j}+\psi_{j}\right)=\phi+\psi$,

(ii) $\sum_{j \in J}\left(c \phi_{j}\right)=c \phi$, where $c$ is any scalar.

Proof. The proof is a direct consequence of Definition 1 and is similar to the proof of the previous lemma.

The fundamental theorem of this paper is the next one which is used to obtain a number of results in Section 3.

Theorem 4. The unordered sum $\sum_{j \in J} \phi_{j}$ is convergent in $X$ if and only if, for any $\epsilon>0$ and $\lambda \in \Lambda$, there exists $J_{0}\left(=J_{0}(\epsilon, \lambda)\right)$ such that $\gamma_{\lambda}\left(S_{K}\right)<\epsilon$ for all $K \in \mathscr{F}$ that are disjoint from $J_{0}$.

Proof. To prove this theorem, we shall use the fact that if $K$ and $L$ are disjoint, finite subsets of $J$, then $S_{K \cup L}=S_{K}+S_{L}$.

Sufficiency. Let $\lambda \in \Lambda$ be arbitrarily fixed, and, for each $n=$ $1,2, \ldots$, choose a finite subset $J_{n}$ of $J$ such that $\gamma_{\lambda}\left(S_{K}\right)<1 / n$ for any $K \in \mathscr{F}$ that is disjoint from $J_{n}$. Replacing $J_{n}$ by $J_{1} \cup$ $J_{2} \cup \cdots \cup J_{n}$, we can assume that $J_{1} \subset J_{2} \subset \cdots$. Then if $m>n$,

$$
\gamma_{\lambda}\left(S_{J_{m}}-S_{J_{n}}\right)=\gamma_{\lambda}\left(S_{J_{m}-J_{n}}\right)<\frac{1}{n}
$$

(as $J_{m}-J_{n}$ is disjoint from $J_{n}$ ).
Hence $\left\{S_{J_{n}}\right\}$ is a Cauchy sequence in $X$, and as we know that $X$ is a complete multinormed space, $\phi=\lim _{n \rightarrow \infty} S_{J_{n}}$ exists. Keeping $n$ fixed, let $m \rightarrow \infty$ in (16) to obtain

$$
\left.\gamma_{\lambda}\left(\phi-S_{J_{n}}\right) \leq \frac{1}{n} \quad \text { (using continuity of } \gamma_{\lambda}\right) .
$$

Now, for any $\epsilon>0$, we can choose $n$ such that $2 / n<\epsilon$. Then, whenever $K \in \mathscr{F}$ contains $J_{n}$, we obtain, from (16) and (17),

$$
\begin{aligned}
\gamma_{\lambda}\left(\phi-S_{K}\right) & =\gamma_{\lambda}\left(\phi-S_{K-J_{n}}-S_{J_{n}}\right) \\
& \leq \gamma_{\lambda}\left(\phi-S_{J_{n}}\right)+\gamma_{\lambda}\left(S_{K-J_{n}}\right) \\
& <\frac{1}{n}+\frac{1}{n}<\epsilon .
\end{aligned}
$$

Thus, $\sum_{j} \phi_{j}$ converges to $\phi$ in $X$.

Necessity. Suppose $\sum_{j \in J} \phi_{j}$ converges to $\phi$ in $X$. Given $\epsilon>0$ and $\lambda \in \Lambda$, we can choose $J_{0}\left(=J_{0}(\epsilon, \lambda)\right)$ such that, whenever $M \in \mathscr{F}$ contains $J_{0}$,

$$
\gamma_{\lambda}\left(S_{M}-\phi\right)<\frac{\epsilon}{2}
$$

Then, for any $K \in \mathscr{F}$ that is disjoint from $J_{0}$, we get

$$
\gamma_{\lambda}\left(\phi-S_{K}-S_{J_{0}}\right)=\gamma_{\lambda}\left(\phi-S_{K \cup J_{0}}\right)<\frac{\epsilon}{2}
$$

(since $K, J_{0}$ are disjoint)

and also

$$
\gamma_{\lambda}\left(\phi-S_{J_{0}}\right)<\frac{\epsilon}{2}
$$

Therefore,

$$
\gamma_{\lambda}\left(S_{K}\right) \leq \gamma_{\lambda}\left(\phi-S_{J_{0}}\right)+\gamma_{\lambda}\left(\phi-S_{K}-S_{J_{0}}\right)<\frac{\epsilon}{2}+\frac{\epsilon}{2}=\epsilon .
$$

Now we state another important result which shows that Definition 1 is equivalent to the usual definition of a convergent series when $J$ is countable.

Lemma 5. Let $J$ be a countable index set. And let the vectors $\left\{\phi_{j}\right\}_{j \in J}$ be summable to $\phi$ in $X$. Then $\phi=\lim _{n \rightarrow \infty} S_{J_{n}}$ for any increasing sequence of finite subsets $J_{n}$ of $J$ such that $\cup_{n} J_{n}=J$.

Proof. Let $\left\{\phi_{j}\right\}_{j \in J}$ be summable to $\phi$. Then from Definition 1, for $\epsilon>0$ and $\lambda \in \Lambda$, there exists $K_{0}\left(=K_{0}(\epsilon, \lambda)\right) \in \mathscr{F}$ such that, whenever $K \in \mathscr{F}$ contains $K_{0}$, we have

$$
\gamma_{\lambda}\left(S_{K}-\phi\right)<\epsilon
$$

As $\left\{J_{n}\right\}$ is an increasing sequence of sets in $\mathscr{F}$ and $\cup_{n} J_{n}=J$, there exists $N \in \mathbb{N}$ such that $K_{0} \subset J_{N}$. Moreover $K_{0} \subset J_{n}$ for all $n \geq N$, and so

$$
\gamma_{\lambda}\left(S_{J_{n}}-\phi\right)<\epsilon \quad \text { whenever } n \geq N \text {. }
$$

Hence the result follows. 
The importance of Lemma 5 is that when $J$ is countable and $\left\{\phi_{j}\right\}_{j \in J}$ is summable to $\phi$ in $X$, then it is often possible to write down explicitly a sequence of partial sums that converge to $\phi$. As a particular case, let $J=\mathbb{Z}$, the set of all integers. Then, we have the following result.

Lemma 6. If $\sum_{j \in \mathbb{Z}} \phi_{j}=\phi$ in $X$, then $\lim _{n \rightarrow \infty} S_{J_{n}}=\phi$, where $J_{n}=\{m \in \mathbb{Z}:|m| \leq n\}$. In this case we can write

$$
\phi=\lim _{n \rightarrow \infty} \sum_{j=-n}^{n} \phi_{j} .
$$

Proof. It follows from Lemma 5.

\section{Orthonormal Expansions in Spaces of Test Functions and Generalized Functions}

In this section, we demonstrate how the theory of summability developed in Section 2 can be used to extend the distributional eigenfunction-expansion theory of Zemanian to the case when we have an uncountable orthonormal basis. As indicated earlier, all orthonormal bases in $L^{2}(I)$ are countable, and so, for our extension to be meaningful, we follow the more general approach introduced in [5] of working with a self-adjoint operator $T$ on an arbitrary Hilbert space $H$. However, unlike [5], where it is assumed that $H$ is separable, we shall allow $H$ to be nonseparable. Throughout we shall assume that $H$ has an (possibly uncountable) orthonormal basis of eigenvectors $\left\{\psi_{j}\right\}_{j \in J}$ of $T$ with corresponding real eigenvalues $\left\{\lambda_{j}\right\}_{j \in J}$. We shall represent the inner product and norm in $H$ by $\|\cdot\|_{H}$ and $(\cdot, \cdot)_{H}$, respectively, and, as before, we shall denote the collection of all finite subsets of $J$ by $\mathscr{F}$.

As discussed in the introduction, $D_{\infty}$ is the Fréchet space:

$$
D_{\infty}:=\bigcap_{k=1}^{\infty} D\left(T^{k}\right)
$$

with multinorm $S=\left\{\beta_{k}\right\}_{k=0}^{\infty}$, where

$$
\beta_{k}(\phi):=\left\|T^{k} \phi\right\|_{H}, \quad k=0,1,2, \ldots ;
$$

see [5, Definition 2.1, Theorem 2.3]. The space of continuous linear functionals on $D_{\infty}$ is denoted by $D_{\infty}^{\prime}$. Convergence in $D_{\infty}^{\prime}$ is defined by the weak ${ }^{*}$ topology which is generated by the multinorm $\left\{\alpha_{\phi}\right\}_{\phi \in D_{\infty}}$, where

$$
\alpha_{\phi}(f):=|(f, \phi)|, \quad f \in D_{\infty}^{\prime}, \phi \in D_{\infty} .
$$

The space $D_{\infty}^{\prime}$ is sequentially complete, and each $\varphi \in H$ generates, uniquely, a continuous linear functional $\tilde{\varphi} \in D_{\infty}^{\prime}$ via the formula

$$
(\widetilde{\varphi}, \phi):=(\phi, \varphi)_{H}, \quad \phi \in D_{\infty}
$$

see [5, Theorems 2.6, 2.7]. As a consequence, we can regard $H$ as a subspace of $D_{\infty}^{\prime}$, and so can write

$$
D_{\infty} \hookrightarrow H \hookrightarrow D_{\infty}^{\prime}
$$

Theorem 7. Let $\phi \in D_{\infty}$. Then

$$
\phi=\sum_{j \in J}\left(\phi, \psi_{j}\right)_{H} \psi_{j},
$$

where the unordered sum is convergent in $D_{\infty}$.

Proof. For any $K \in \mathscr{F}$, we can use the linearity of $T$ to write

$$
\begin{aligned}
& \beta_{k}\left(\sum_{j \in K}\left(\phi, \psi_{j}\right)_{H} \psi_{j}-\phi\right) \\
& =\left\|\sum_{j \in K}\left(\phi, \psi_{j}\right)_{H} T^{k} \psi_{j}-T^{k} \phi\right\|_{H} \\
& =\left\|\sum_{j \in K}\left(\phi, \psi_{j}\right)_{H} \lambda_{j}^{k} \psi_{j}-T^{k} \phi\right\|_{H} \\
& =\left\|\sum_{j \in K}\left(\phi, T^{k} \psi_{j}\right)_{H} \psi_{j}-T^{k} \phi\right\|_{H} \\
& =\left\|\sum_{j \in K}\left(T^{k} \phi, \psi_{j}\right)_{H} \psi_{j}-T^{k} \phi\right\|_{H} .
\end{aligned}
$$

Since, for each $k \in \mathbb{N}_{0},\left\{\left(T^{k} \phi, \psi_{j}\right)_{H} \psi_{j}\right\}_{j \in J}$ is summable to $T^{k} \phi$ in $H$, it follows that, for $\epsilon>0$ and $k \in \mathbb{N}_{0}$, there exists $J_{0}(=$ $\left.J_{0}(\epsilon, k)\right) \in \mathscr{F}$ such that

$$
\left\|\sum_{j \in K}\left(T^{k} \phi, \psi_{j}\right)_{H} \psi_{j}-T^{k} \phi\right\|_{H}<\epsilon
$$

for all $K \in \mathscr{F}$ that contain $J_{0}$. But then

$$
\beta_{k}\left(\sum_{j \in K}\left(\phi, \psi_{j}\right)_{H} \psi_{j}-\phi\right)<\epsilon
$$

for all $K \in \mathscr{F}$ that contain $J_{0}$. Hence $\left\{\left(\phi, \psi_{j}\right)_{H} \psi_{j}\right\}_{j \in J}$ is summable in $D_{\infty}$ to $\phi$.

It is also possible to characterize the unconditional convergence of sums of the form $\sum_{j \in J} a_{j} \psi_{j}$, where $a_{j} \in \mathbb{C}$ for all $j \in J$, in the following way.

Theorem 8. Let $\left\{a_{j}\right\}_{j \in J}$ be a family of scalars. Then $\left\{a_{j} \psi_{j}\right\}_{j \in J}$ is summable to some $\phi \in D_{\infty}$ if and only if $\left\{\left|a_{j}\right|^{2}\left|\lambda_{j}\right|^{2 k}\right\}_{j \in J}$ is summable in $\mathbb{R}$ for each $k \in \mathbb{N}_{0}$, in which case $a_{j}=\left(\phi, \psi_{j}\right)_{H}$ for all $j$.

Proof. For each $K \in \mathscr{F}$, let $\mathcal{S}_{K}=\sum_{j \in K} a_{j} \psi_{j}$. Then for each $\beta_{k}$, we obtain

$$
\left[\beta_{k}\left(\mathcal{S}_{K}\right)\right]^{2}=\left\|\sum_{j \in K} a_{j} T^{k} \psi_{j}\right\|_{H}^{2}=\sum_{j \in K}\left|a_{j}\right|^{2} \lambda_{j}^{2 k} .
$$

Suppose that $\left\{\left|a_{j}\right|\left|\lambda_{j}\right|^{2 k}\right\}_{j \in J}$ is summable in $\mathbb{R}$ for each $k \epsilon$ $\mathbb{N}_{0}$, and let $\epsilon>0$. Then by Theorem 4 , there exists $J_{0} \in \mathscr{F}$ 
such that $\sum_{j \in K} \lambda_{j}^{2 k}\left|a_{j}\right|^{2}<\epsilon^{2}$ for any $K \in \mathscr{F}$ that is disjoint from $J_{0}$. But then $\beta_{k}\left(\mathcal{S}_{K}\right)<\epsilon$ for any such $K$. Since this can be done for each $\beta_{k},\left\{a_{j} \psi_{j}\right\}_{j \in J}$ is summable in $D_{\infty}$.

Conversely, if $\left\{a_{j} \psi_{j}\right\}_{j \in J}$ is summable in $D_{\infty}$, then again, by Theorem 4, for any $\epsilon>0$ and $k \in \mathbb{N}_{0}$, there exists $J_{0} \in \mathscr{F}$ such that $\beta_{k}\left(\mathcal{S}_{K}\right)<\epsilon$ for every $K \in \mathscr{F}$ disjoint from $J_{0}$. Hence, from (35),

$$
\sum_{j \in K} \lambda_{j}^{2 k}\left|a_{j}\right|^{2}<\epsilon^{2}
$$

for any such $K$, which implies that $\left\{\left|a_{j}\right|^{2}\left|\lambda_{j}\right|^{2 k}\right\}_{j \in J}$ is summable in $\mathbb{R}$ for each $k \in \mathbb{N}_{0}$.

The final statement in the theorem follows from the fact that, for each fixed $l \in J$,

$$
\left(\phi, \psi_{l}\right)_{H}=\sum_{j \in J} a_{j} \bar{b}_{j},
$$

where $b_{l}=1$ and $b_{j}=0, j \neq l$; see [9, Theorem 6.26].

Corresponding results on the weak $^{*}$ convergence of eigenvector expansions in the space $D_{\infty}^{\prime}$ can also be established. Once again, these can be regarded as natural extensions of the theory developed in [3, Chapter 9] to the case of an index set $J$ which need not be countable. First, note that $\left\{\widetilde{\psi}_{j}\right\}_{j \in J}$ can be regarded as a collection of "regular generalized eigenvectors" in $D_{\infty}^{\prime}$. As the following theorem shows, each $f \in D_{\infty}^{\prime}$ can be expressed as a weak ${ }^{*}$-convergent Fourier series in terms of this collection of regular generalized eigenvectors.

Theorem 9. Each $f \in D_{\infty}^{\prime}$ can be expressed in the form

$$
f=\sum_{j \in J}\left(f, \psi_{j}\right) \widetilde{\psi}_{j},
$$

where the unordered sum is convergent in $D_{\infty}^{\prime}$.

Proof. Let $\epsilon>0$ and $\phi \in D_{\infty}$. Then, with $S_{K}=\sum_{j \in K}\left(f, \psi_{j}\right) \tilde{\psi}_{j}$, where $K \in \mathscr{F}$, we have

$$
\begin{aligned}
\alpha_{\phi}\left(S_{K}-f\right) & =\left|\left(S_{K}-f, \phi\right)\right| \\
& =\left|\left(\sum_{j \in K}\left(f, \psi_{j}\right) \tilde{\psi}_{j}, \phi\right)-(f, \phi)\right| \\
& =\left|\left(f, \sum_{j \in K}\left(\phi, \psi_{j}\right)_{H} \psi_{j}-\phi\right)\right| \\
& \leq M_{f} \sum_{r=0}^{k_{f}} \alpha_{r}\left(\sum_{j \in K}\left(\phi, \psi_{j}\right)_{H} \psi_{j}-\phi\right)
\end{aligned}
$$

(by continuity of $f$; see [3, Theorem 1.8-1]).

Since $\sum_{j \in J}\left(\phi, \psi_{j}\right)_{H} \psi_{j}=\phi$ in $D_{\infty}$ and there are only a finite number of seminorms $\alpha_{r}$, it follows that there exists $J_{0} \in$ $\mathscr{F}$ such that

$$
\alpha_{\phi}\left(S_{K}-f\right)<\epsilon,
$$

for all $K \in \mathscr{F}$ that contain $J_{0}$. Hence

$$
f=\sum_{j \in J}\left(f, \psi_{j}\right) \widetilde{\psi}_{j} .
$$

Theorem 10. Let the null space $N(T)$ of $T$ be finitedimensional and let $\left\{b_{j}\right\}_{j \in J} \subset \mathbb{C}$. Then

$$
\sum_{j \in J} b_{j} \widetilde{\psi}_{j}=f
$$

in $\mathscr{A}^{\prime}$ if there exists $q \in \mathbb{N}_{0}$ such that

$$
\sum_{j \in J: \lambda_{j} \neq 0} \lambda_{j}^{-2 q}\left|b_{j}\right|^{2}
$$

is convergent in $\mathbb{R}$. Moreover, in this case $b_{j}=\left(f, \psi_{j}\right)$ for each $j$.

Proof. Let $J^{\prime}=\left\{j \in J: \lambda_{j} \neq 0\right\}$ and, for each finite subset $K^{\prime}$ of $J^{\prime}$, let

$$
S_{K^{\prime}}=\sum_{j \in K^{\prime}} b_{j} \widetilde{\psi}_{j}
$$

By the convergence assumption and Theorem 4 , it follows that, for $\epsilon>0$, there exists a finite subset $J_{0}^{\prime}$ of $J^{\prime}$ such that

$$
\sum_{j \in K^{\prime}} \lambda_{j}^{-2 q}\left|b_{j}\right|^{2}<\epsilon
$$

for all finite subsets $K^{\prime}$ of $J^{\prime}$ disjoint from $J_{0}^{\prime}$. Also, from Theorem 8 ,

$$
\sum_{j \in J} \lambda_{j}^{2 q}\left|\left(\phi, \psi_{j}\right)_{H}\right|^{2}<\infty
$$

Hence there exists a finite subset $J_{1}^{\prime}$ of $J^{\prime}$ such that

$$
\sum_{j \in K_{1}^{\prime}} \lambda_{j}^{2 q}\left|\left(\phi, \psi_{j}\right)_{H}\right|^{2}<\epsilon
$$

for all finite subsets $K_{1}^{\prime}$ of $J^{\prime}$ disjoint from $J_{1}^{\prime}$. Let $J_{2}^{\prime}=J_{0}^{\prime} \cup J_{1}^{\prime}$. Then, for any finite subset $K^{\prime}$ of $J^{\prime}$ that is disjoint from $J_{2}^{\prime}$, we have

$$
\begin{aligned}
& \alpha_{\phi}\left(S_{K^{\prime}}\right) \\
& =\left|\left(\sum_{j \in K^{\prime}} b_{j} \widetilde{\psi}_{j}, \phi\right)\right|=\left|\sum_{j \in K^{\prime}} b_{j}\left(\phi, \psi_{j}\right)_{H}\right| \\
& =\left|\sum_{j \in K^{\prime}} b_{j}\left(\lambda_{j}^{q} \phi, \lambda_{j}^{-q} \psi_{j}\right)_{H}\right| \leq \sum_{j \in K^{\prime}}\left|b_{j} \lambda_{j}^{-q}\left(\lambda_{j}^{q} \phi, \psi_{j}\right)_{H}\right| \\
& \quad \leq\left(\sum_{j \in K^{\prime}}\left|b_{j} \lambda_{j}^{-q}\right|^{2} \sum_{j \in K^{\prime}}\left|\lambda_{j}^{q}\left(\phi, \psi_{j}\right)_{H}\right|^{2}\right)^{1 / 2}<\epsilon .
\end{aligned}
$$


Therefore $\sum_{j \in J^{\prime}} b_{j} \widetilde{\psi}_{j}$ is convergent in $\mathscr{A}^{\prime}$, which implies that $\sum_{j \in J} b_{j} \widetilde{\psi}_{j}$ is also summable in $\mathscr{A}^{\prime}$ since $N(T)$ is finitedimensional.

To complete the proof, we use the fact that since $f$ is a continuous linear functional, we have

$$
\begin{aligned}
\left(f, \psi_{l}\right) & =\left(\sum_{j \in J} b_{j} \widetilde{\psi}_{j}, \psi_{l}\right) \\
& =\sum_{j \in J} b_{j}\left(\widetilde{\psi}_{j}, \psi_{l}\right)=\sum_{j \in J} b_{j}\left(\psi_{l}, \psi_{j}\right)_{H}=b_{l} .
\end{aligned}
$$

\section{Almost-Periodic Generalized Functions}

To illustrate the theory of expansions of test functions and generalized functions in terms of an uncountable basis of eigenfunctions, we examine the differential operator $-i D$ on the Hilbert space $H$ of $L^{2}$ almost-periodic functions. As explained in [9, Example 6.19], to obtain this space we consider first the vector space $V$ of quasiperiodic functions $\eta: \mathbb{R} \rightarrow \mathbb{C}$ of the form

$$
\eta(x)=\sum_{k=1}^{n} a_{k} e^{i \omega_{k} x},
$$

where $n \in \mathbb{N}, a_{k} \in \mathbb{C}$ and $\omega_{k} \in \mathbb{R}$ are arbitrary constants. This vector space becomes a pre-Hilbert space when we define on it the inner product

$$
(\eta, \xi)_{H}:=\lim _{R \rightarrow \infty} \frac{1}{2 R} \int_{-R}^{R} \eta(x) \overline{\xi(x)} d x
$$

The Hilbert space $H$ is then obtained as the completion of this pre-Hilbert space. Elements in $H$ are equivalence classes of functions of the form

$$
\phi(x)=\sum_{k=1}^{\infty} a_{k} e^{i \omega_{k} x},
$$

where $\sum_{k=1}^{\infty}\left|a_{k}\right|^{2}<\infty$ and the sum in (52) converges with respect to the norm induced by the inner product (51). The set of functions

$$
\left\{\psi_{\omega}\right\}_{\omega \in \mathbb{R}}, \quad \psi_{\omega}(x):=e^{i \omega x},
$$

is an uncountable orthonormal basis for $H$, showing that $H$ is not separable. Note that each $\psi_{\omega}$ can be regarded as an eigenfunction of $-i D$, defined on the domain $V \subset H$, with corresponding eigenvalue $\omega$.

To enable a Fréchet space $D_{\infty}$ of test functions and associated space of generalized functions $D_{\infty}^{\prime}$ to be constructed, we require a self-adjoint extension $T$ of $-i D$. The fact that the set in (53) is an orthonormal basis for $H$ enables us to define $T$ in an obvious manner as

$$
\begin{aligned}
& T \phi:=\sum_{\omega \in \mathbb{R}} \omega\left(\phi, \psi_{\omega}\right)_{H} \psi_{\omega}, \\
& D(T):=\left\{\phi \in H: \sum_{\omega \in \mathbb{R}}\left|\omega\left(\phi, \psi_{\omega}\right)_{H}\right|^{2}<\infty\right\} .
\end{aligned}
$$

Note that, for any given $\phi \in D(T)$, only countably many terms in the expansions in (54) will be nonzero.

The general theory developed earlier now leads immediately to the following expansion result for generalized functions in $D_{\infty}^{\prime}$.

Theorem 11. Let $D_{\infty}^{\prime}$ be the space of generalized functions constructed around the self-adjoint extension of -iD given by (54). Then each $f \in D_{\infty}^{\prime}$ can be expressed in the form

$$
f=\sum_{\omega \in \mathbb{R}}\left(f, \psi_{\omega}\right) \tilde{\psi}_{\omega}
$$

where $\psi_{\omega}(x)=\exp (i \omega x)$ and the unordered sum is convergent in $D_{\infty}^{\prime}$.

In this particular case, we can interpret $D_{\infty}^{\prime}$ as a space of almost-periodic generalized functions.

\section{Conclusion}

A theory of summability has been developed and applied to obtain important convergence results of an orthonormal set $\left\{\phi_{j}\right\}_{j \in J}$ in suitably constructed spaces of test functions and generalized functions. As a particular case, a space of almostperiodic generalized functions has been produced, in which each generalized function can be expanded in terms of an uncountable orthonormal basis of exponential functions.

\section{References}

[1] K. N. Khan, W. Lamb, and A. C. McBride, "Fractional transformations of generalized functions," Integral Transforms and Special Functions, vol. 20, no. 5-6, pp. 471-490, 2009.

[2] K. N. Khan, W. Lamb, and A. C. McBride, "Fractional calculus of periodic distributions," Fractional Calculus and Applied Analysis, vol. 14, no. 2, pp. 260-283, 2011.

[3] A. H. Zemanian, Generalized Integral Transformations, Interscience Publishers, New York, NY, USA, 1968.

[4] D. Judge, “On Zemanian's distributional eigenfunction transforms," Journal of Mathematical Analysis and Applications, vol. 34, pp. 187-201, 1971.

[5] W. Lamb and D. F. McGhee, "Spectral theory and functional calculus for operators on spaces of generalized functions," Journal of Mathematical Analysis and Applications, vol. 163, no. 1, pp. 238-260, 1992.

[6] R. S. Pathak, "Orthogonal series representations for generalized functions," Journal of Mathematical Analysis and Applications, vol. 130, no. 2, pp. 316-333, 1988.

[7] S. Pilipović, "Generalization of Zemanian spaces of generalized functions which have orthonormal series expansions," SIAM Journal on Mathematical Analysis, vol. 17, no. 2, pp. 477-484, 1986.

[8] T. Kato, Perturbation Theory for Linear Operators, Springer, Berlin, Germany, 1966.

[9] J. K. Hunter and B. Nachtergaele, Applied Analysis, World Scientific, Singapore, 2001. 
[10] J. D. Pryce, Basic Methods of Linear Functional Analysis, Hutchinson, London, UK, 1973.

[11] R. S. Pathak, Integral Transforms of Generalized Functions and their Applications, Gordon and Breach, Amsterdam, The Netherlands, 1997. 


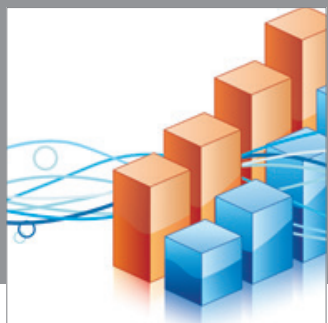

Advances in

Operations Research

mansans

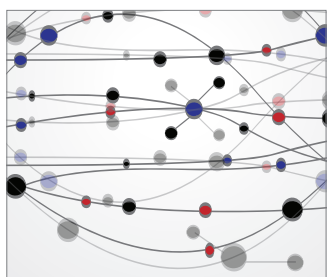

The Scientific World Journal
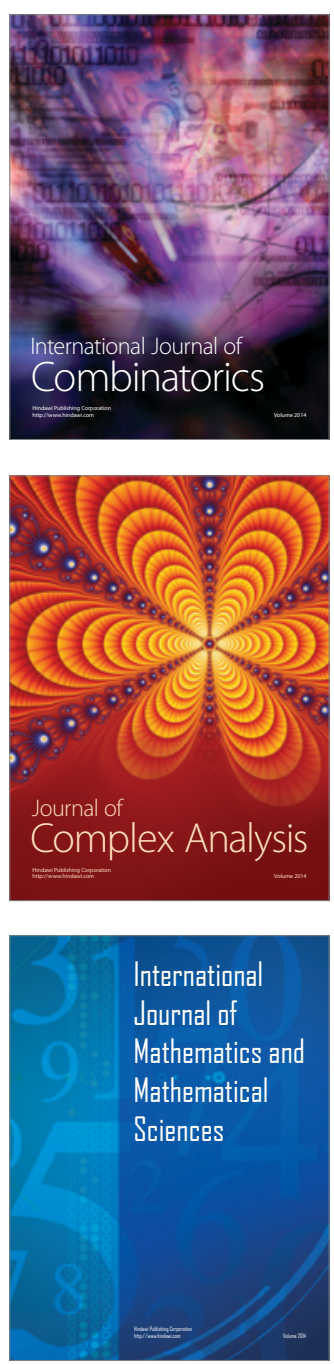
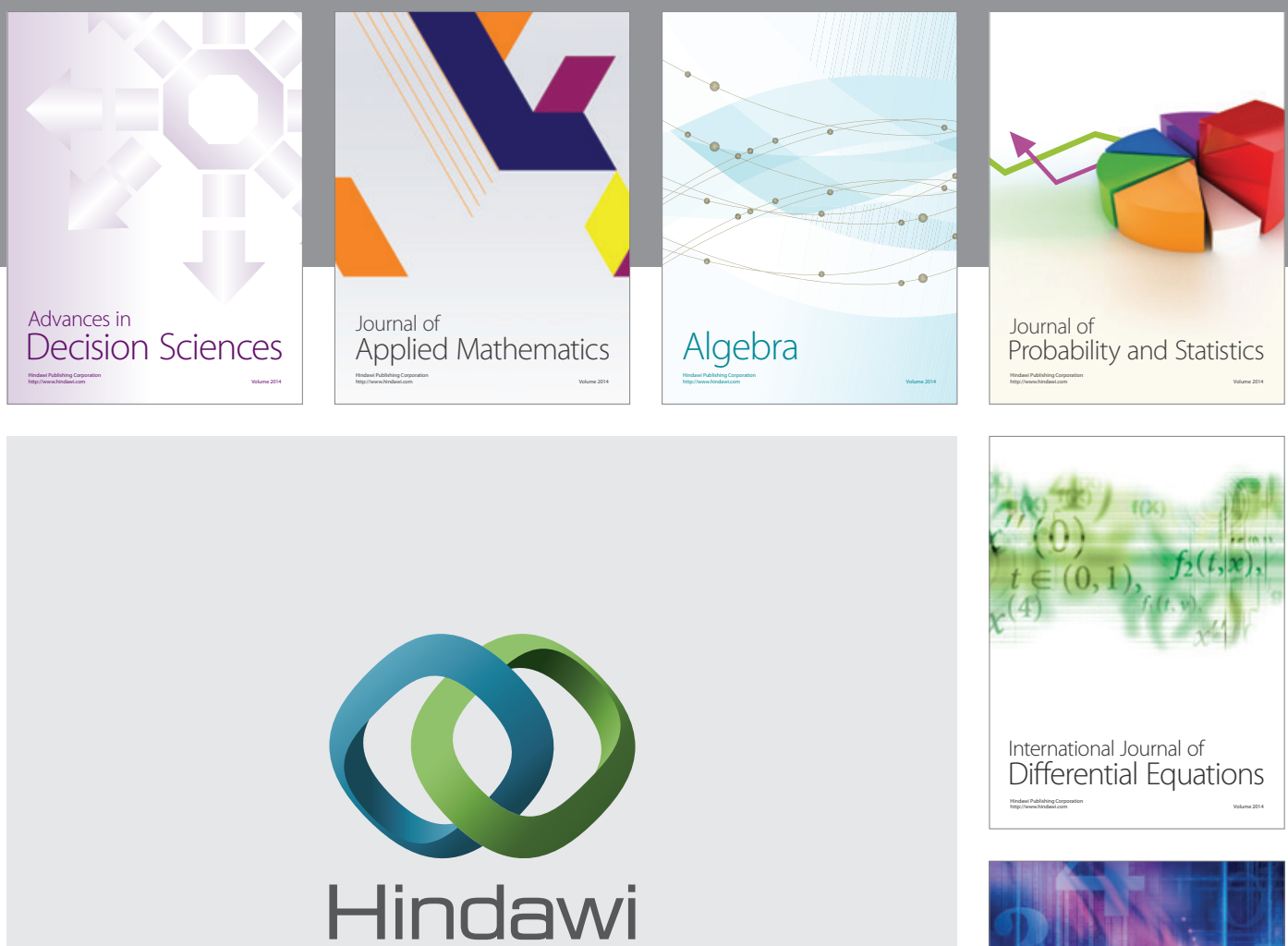

Submit your manuscripts at http://www.hindawi.com
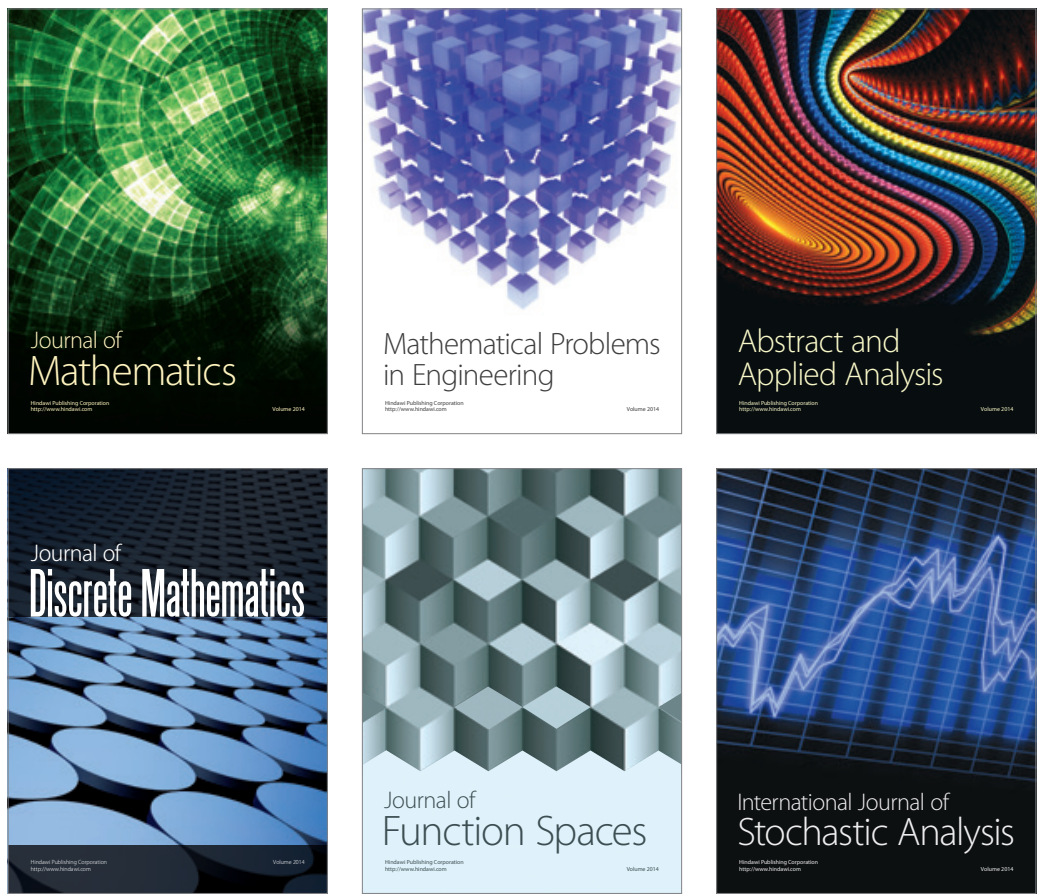

Journal of

Function Spaces

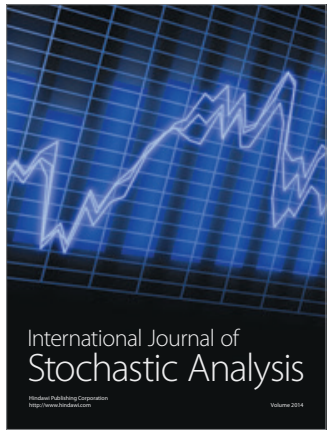

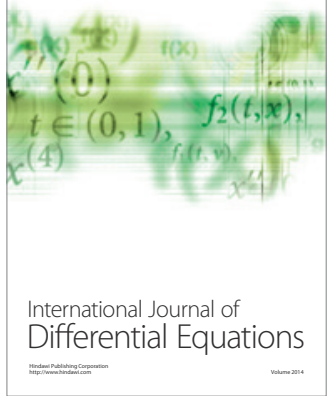
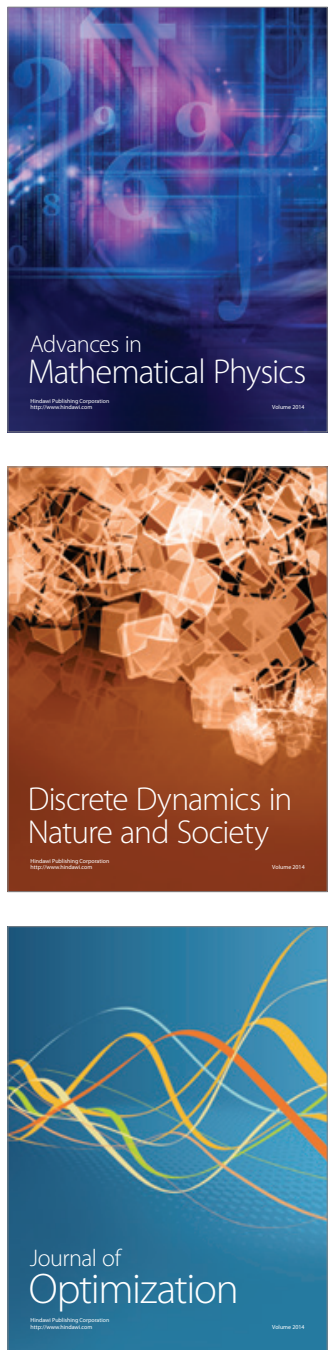